OPEN ACCESS

Edited by:

Bing $X u$,

Xiamen University, China

Reviewed by: Wei Wu,

Central South University, China

Ravi Manoharan,

University of Madras, India Kun Zhou,

Boston Children's Hospital, United States

${ }^{*}$ Correspondence: Sen Zhang zs0771@126.com Liang Kang

kangl@mail.sysu.edu.cn

${ }^{\dagger}$ These authors have contributed equally to this work

Specialty section:

This article was submitted to Molecular and Cellular Oncology, a section of the journal

Frontiers in Oncology

Received: 07 September 2020 Accepted: 08 April 2021 Published: 07 May 2021

Citation:

Huang L, Luo S, Zhang X, Cai Y, Xue F Hu H, Zeng Z, Lin T, Wang F, Wang W, Zhang S and Kang L (2021) Distinct Genomic Landscape of Colorectal

Mucinous Carcinoma Determined via Comprehensive Genomic Profiling: Steps to a New Treatment Strategy.

Front. Oncol. 11:603564. doi: 10.3389/fonc.2021.603564

\section{Distinct Genomic Landscape of Colorectal Mucinous Carcinoma Determined via Comprehensive Genomic Profiling: Steps to a New Treatment Strategy}

\author{
Liang Huang ${ }^{1,2,3 \dagger}$, Shuanglin Luo ${ }^{1,2,3 \dagger}$, Xingwei Zhang ${ }^{1,2,3}$, Yonghua Cai ${ }^{1,2,3}$, \\ Fangqin Xue ${ }^{4}$, Huanxin Hu ${ }^{1,2,3}$, Ziwei Zeng ${ }^{1,2,3}$, Tengjiao Lin $^{5}$, Fei Wang ${ }^{5}$, Weifeng Wang ${ }^{5}$, \\ Sen Zhang ${ }^{6 *}$ and Liang Kang ${ }^{1,2,3 *}$ \\ ${ }^{1}$ Department of Colorectal Surgery, The Sixth Affiliated Hospital of Sun Yat-Sen University, Guangzhou, China, ${ }^{2}$ Guangdong \\ Institute of Gastroenterology, The Sixth Affiliated Hospital of Sun Yat-Sen University, Guangzhou, China, ${ }^{3}$ Guangdong \\ Provincial Key Laboratory of Colorectal and Pelvic Floor Diseases, The Sixth Affiliated Hospital of Sun Yat-Sen University, \\ Guangzhou, China, ${ }^{4}$ Department of Gastrointestinal Surgery, Fujian Provincial Hospital, Fuzhou, China, ${ }^{5}$ Department of \\ Research and Development, OrigiMed, Shanghai, China, ${ }^{6}$ Department of Colorectal Surgery, The First Affiliated Hospital of \\ Guangxi Medical University, Nanning, China
}

Colorectal mucinous carcinoma $(\mathrm{MC})$ is associated with inferior prognosis and response to treatment compared to adenocarcinoma (AC). The molecular landscapes of MC and adenocarcinoma with mucous composition (AMC) are not well-defined. We aimed to describe the genomic landscape of $\mathrm{MC}$ and $\mathrm{AMC}$ in a large colorectal cancer cohort. Tumor samples from patients with $\mathrm{MC}, \mathrm{AMC}$, or $\mathrm{AC}$ were analyzed using next-generation sequencing. $\mathrm{MC}$ had a molecular signature distinct from that of $\mathrm{AC}$; genomic features were similar between $\mathrm{AMC}$ and $\mathrm{MC}$ but not between $\mathrm{AMC}$ and $\mathrm{AC}$. HER2 amplification and TP53 and APC mutation rates were lower, whereas SMAD4, PIK3CA, ACVR2A, KMT2D, LRP1, TGFBR2, GRIN2A, BRAF V600E, PTEN, and BRCA2 mutation rates were higher in MC than in AC. The mutation frequencies in MAPK, PI3K, and TGF- $\beta$ pathways were higher, whereas those of cell cycle proteins and Wnt were lower in MC and AMC than in AC. The proportion of hypermutated tumors was significantly higher in $\mathrm{MC}$ and AMC than in AC. As MC has a distinct molecular signature from AC, immunotherapy can be potentially applied in treating MC. Similar molecular profiles of AMC and MC suggest that treatment strategies for $\mathrm{MC}$, but not $\mathrm{AC}$, can be used for $\mathrm{AMC}$ treatment.

Keywords: colorectal cancer, mucinous adenocarcinoma, adenocarcinoma with mucous composition, next-generation sequencing, hypermutated tumor

\section{INTRODUCTION}

According to the 2018 global cancer statistics released by the International Cancer Research Institute of the World Health Organization (WHO), colorectal cancer (CRC) has the third highest incidence rate and second highest mortality rate, and an increasing annual prevalence rate $(1,2)$. According to the WHO classification, mucinous carcinoma (MC) is a distinct pathological CRC subtype, with a substantial mucous component of more than $50 \%$ of the tumor volume, and accounts for $10-15 \%$ of all CRC cases $(3,4)$. MC constitutes a histological subtype with poor differentiation potential and is a predictive factor for poor prognosis $(5,6)$. 
MC is clinically more prevalent among women, frequently located in the proximal colon, and associated with young age, high malignancy grade, tumor infiltration, lymph node metastasis, and peritoneal metastasis $(4,7)$. Compared with adenocarcinoma not otherwise specified (AC), patients with $\mathrm{MC}$ are reportedly less responsive to neoadjuvant radiotherapy and chemotherapy (8). The efficacy of first-line chemotherapy with oxaliplatin or irinotecan is lower among patients with advanced MC than among those with AC. Furthermore, patients with metastatic MC do not benefit from treatment with anti-epidermal growth factor (EGFR) monoclonal antibodies, even in cases with wild-type RAS and BRAF (9). Therefore, it is important to investigate the molecular characteristics of colorectal MC in detail and explore a more effective treatment strategy.

Colorectal MC has unique molecular characteristics. Most early studies focused on protein expression levels and reported that MUC2 and MUC5AC are upregulated in MC tumors (10, 11). Recent genomic analyses have reported that colorectal MC has a higher mutation frequency in Ras/MAPK and PI3K/Akt/ mTOR pathways in MC than in AC, with a higher incidence of microsatellite instability (MSI), which is potentially associated with Lynch syndrome and the CpG island methylator phenotype (4). However, owing to limitations in detection technology, previous studies have not revealed the genetic landscape of $\mathrm{MC}$, including comprehensive genomic characteristics, pathway analyses, and biomarkers for immunotherapy. The fraction of mucous composition varies substantially among Colorectal Cancers. Prior studies confirm that the variation of mucous composition in CRC is associated with distinct molecular and clinical features $(12,13)$. However, adenocarcinomas with relatively low mucous composition (less than 50\%, also known as AMC) are usually diagnosed and treated as AC. The somatic mutational landscape of this unique subgroup is less known and the best clinical management of AMC needs to be addressed in the light of the mutational background (4).

In this study, we aimed to perform comprehensive targeted next-generation sequencing (NGS) to detect the two pathological subtypes of $\mathrm{CRC}, \mathrm{MC}$ and $\mathrm{AMC}$, and gain deep insights into their molecular characteristics, through the evaluation of the landscape of genetic alterations, pathway analysis, and analysis of biomarkers for immunotherapy to provide a molecular basis for the establishment of a precise treatment strategy for MC and AMC.

\section{MATERIALS AND METHODS}

\section{Patients and Tumor Selection}

Tumor specimens of patients with CRC involved in this study from January 2018 to September 2019 were sent for NGS analysis. Of 2,115 patients with CRC, 1,226 with a confirmed pathological diagnosis of $\mathrm{MC}, \mathrm{AMC}$, or $\mathrm{AC}$ were selected and recruited. Patients with an uncertain diagnosis of the pathological subtype or those with other special pathological subtypes, such as signet-ring cell carcinoma, undifferentiated carcinoma, and squamous cell carcinoma, were excluded. Of 2,115 patients with CRC, 1,226 with a confirmed pathological diagnosis of $\mathrm{MC}, \mathrm{AMC}$, or AC were selected and recruited. Patients with an uncertain diagnosis of the pathological subtype or those with other special pathological subtypes, such as signetring cell carcinoma, undifferentiated carcinoma, and squamous cell carcinoma, were excluded. MC was defined as extracellular mucus secretion accounting for $>50 \%$ of the tumor volume. AMC was defined that accounted for $\leq 50 \%$. And AC was defined as tumor with no extracellular mucus secretion. All tumor tissues were assessed independently by two experienced pathologists before sample disposal to pathologically confirm the diagnoses.

This study was approved by the Institution Review Board of the Sixth Affiliated Hospital of Sun Yat-sen University in accordance with the Declaration of Helsinki. Written informed consent was obtained from all enrolled patients.

\section{NGS Analysis}

NGS analysis was carried out at OrigiMed (Shanghai, China), a College of American Pathologists-accredited and Clinical Laboratory Improvement Amendments-certified laboratory, using a 450-gene comprehensive assay (14). At least $50 \mathrm{ng}$ of DNA was extracted from each $40 \mathrm{~mm}$ formalin-fixed paraffinembedded (FFPE) tumor sample using a DNA Extraction Kit (QIAamp DNA FFPE Tissue Kit) in accordance with the manufacturer's protocols. This panel encompassed all coding exons of 450 cancer-related genes and 64 selected introns of 39 genes that are frequently rearranged in solid tumors. Furthermore, the probe density was increased to ensure high capture efficiency in the conservatively low-read-depth regions. Peripheral blood was sampled from each patient as the normal control sample for genomic profiling. The genes were captured and sequenced with a mean coverage of $900 \times$ for FFPE samples and $300 \times$ for matched blood samples using an Illumina NextSeq 500 Platform (Illumina Incorporated, San Diego, CA, USA).

\section{Genetic Analysis}

All types of genetic alterations, including single-nucleotide variant $(\mathrm{SNV})$, short and long indels, copy number alterations (CNAs), and gene rearrangement, were called using a suite of bioinformatics pipelines. Analysis of SNVs and indels began with the alignment of raw reads to the human genome reference sequence (hg19) with the Burrows-Wheeler Aligner (v0.62; BWA, Cambridge, MA, USA), followed by polymerase chain reaction (PCR) duplicates removal using MarkDuplicates algorithm from Picard (version 1.47; Cambridge, MA, USA). Local realignment and base quality recalibration for SNVs were performed using GATK (v3.1-1; Cambridge, MA, USA) and subsequently called by MUTECT (v1.7; Cambridge, MA, USA). The CNAs included: (1) amplification, defined as an increase in the number of gene segment copies by $\geq 8$, and (2) homozygous deletion, defined as decrease of complete loss of gene segment copies in samples with $20 \%$ purity. To identify these alterations, tumor cellularity was estimated by allele frequencies of sequenced single-nucleotide polymorphisms (SNPs). For detection of gene rearrangement, aligned reads with abnormal insert size of 2,000 or zero bp were collected and used as 
discordant reads, that is, paired-end reads that could not be closely mapped to a genome reference, with each read of paired reads aligned to the same chromosomes or different chromosomes. Originally, the discordant reads with the distance less than $500 \mathrm{bp}$ formed clusters were further assembled by fermi-lite to identify potential rearrangement breakpoints. The breakpoints were double confirmed by BLAT, and the resulting chimeric gene candidates were annotated. For germline mutations, common single nucleotide polymorphisms, defined as those from the dbSNP database (Version 147), at a frequency of more than $1.5 \%$ from the Exome Sequencing Project 6500 (ESP6500), or at a frequency of more than $1.5 \%$ from the 1000 Genomes Project, were excluded. Furthermore, the variant allele frequency was adjusted with tumor purity estimated using FACETS.

\section{Tumor Mutational Burden (TMB) and MSI}

The TMB was estimated using the method of Chalmers et al. (15). In brief, the somatic, coding, base substitution, and short indel mutations were enumerated. Driver mutations and germline alterations in the dbSNP database were not enumerated. The TMB was determined by dividing the total number of mutations by the size of the coding region. The MSI status was determined in all cases. Based on the MSI score, samples were classified as MSIhigh (MSI-H) and microsatellite stable (MSS).

\section{Statistical Analyses}

Qualitative variables were assessed using Fisher's exact test. Normally distributed quantitative data were analyzed using the $t$-test and non-normally distributed data were analyzed using the Wilcoxon rank test. All tests were two-tailed and significance was defined as a $P$ value less than 0.05 . All statistical analyses were performed using $\mathrm{R}$ software (Version 3.4.2).

\section{RESULTS}

\section{Clinical Characteristics}

We defined MC as adenocarcinoma with mucous composition greater than $50 \%$ and $\mathrm{AC}$ as adenocarcinoma with no mucous composition. Adenocarcinoma with mucous composition but less than $50 \%$ is called AMC. Table $\mathbf{1}$ summarizes the characteristics of the patients. In total, 1,226 patients with CRC were enrolled in the study and divided into three categories by histological subtype: MC (10.5\%), and AMC (8.2\%), and AC (81.3\%). The median age of patients with $\mathrm{MC}$ was less than that of patients with AC (56 vs. 59 years, $P=0.037$ ), and the incidence of MC in the right colon was higher than that of $\mathrm{AC}(41.9$ vs. $24.2 \%, P<0.001)$. Patients with MC accounted for a larger proportion of patients with stage III CRC (44.2\%) than AC $(29.9 \%, P<0.001)$ and AMC (29.0\%, $P=0.091)$; but for AMC, the difference is only marginally significant. Furthermore, AMC was significantly more common in the right colon than $\mathrm{AC}$ was (50 vs. $24.2 \%, P<0.001)$. No significant differences were observed between AMC and AC with respect to other clinical features.

\section{Comparison of Common Gene Mutations Among MC, AMC, and AC}

Comprehensive targeted NGS revealed that the top 10 prevalent mutations in MC were KRAS (55.8\%), TP53 (53.5\%), APC (46.5\%), SMAD4 (34.1\%), ACVR2A (28.7\%), PIK3CA (28.7\%), KMT2D (22.5\%), LRP1 (21.7\%), TGFBR2 (20.2\%), and ARID1A (19.4\%)

(Figure 1A). In general, the mutation profile of MC was different from that of AC (Figure 1B); however, the mutation profiles of MC and AMC did not differ significantly (data not shown). Among the commonly mutated genes in CRC, TP53 (53.5 vs. 79.5\%, $P<0.001$ ) and $A P C(46.5$ vs. $75.1 \%, P<0.001)$ displayed a significantly lower mutation rate, whereas SMAD4 (34.1 vs. 19.1\%, $P<0.001$ ), PIK3CA

TABLE 1 | Patient and tumor characteristics.

\begin{tabular}{|c|c|c|c|c|c|c|}
\hline Characteristics & MCN = $129(\%)$ & $A M C N=100(\%)$ & $A C N=997(\%)$ & $P$ value $\mathrm{MC}$ vs. AC & $P$ value $A M C$ vs. AC & $P$ value $M C$ vs. AMC \\
\hline Gender & & & & 1.000 & 0.134 & 0.227 \\
\hline Female & $50(38.8)$ & $47(47)$ & $390(39.1)$ & & & \\
\hline Male & $79(61.2)$ & $53(53)$ & $607(60.9)$ & & & \\
\hline Age & & & & 0.037 & 0.399 & 0.043 \\
\hline Median & 56 & 62 & 59 & & & \\
\hline Range & $17-86$ & 28-82 & $16-96$ & & & \\
\hline Primary Tumor Site & & & & $<0.001$ & $<0.001$ & 0.016 \\
\hline Left colon & 55 (42.6) & $25(25)$ & $320(32.1)$ & & & \\
\hline Right colon & $54(41.9)$ & $50(50)$ & $241(24.2)$ & & & \\
\hline Rectum & $20(15.5)$ & $24(24)$ & $427(42.8)$ & & & \\
\hline NA & $0(0)$ & $1(1)$ & $9(0.9)$ & & & \\
\hline Stage at diagnosis ${ }^{a}$ & & & & $<0.001$ & 0.133 & 0.091 \\
\hline Stage I & $3(2.3)$ & $5(5)$ & $58(5.8)$ & & & \\
\hline Stage II & $40(31)$ & $33(33)$ & 232 (23.3) & & & \\
\hline Stage III & 57 (44.2) & $29(29)$ & $298(29.9)$ & & & \\
\hline Stage IV & $27(20.9)$ & $32(32)$ & $367(36.8)$ & & & \\
\hline NA & $2(1.6)$ & $1(1)$ & $42(4.2)$ & & & \\
\hline Sample Source & & & & 0.415 & 0.622 & 0.177 \\
\hline Primary lesion & $126(97.6)$ & $100(100)$ & $984(98.7)$ & & & \\
\hline Metastatic lesion & $3(2.4)$ & $0(0)$ & $13(1.3)$ & & & \\
\hline
\end{tabular}

aStage at diagnosis based on AJCC (8th edition). MC, mucinous carcinoma; AMC, adenocarcinoma with mucous composition; AC, adenocarcinoma; AJCC, American Joint Committee on Cancer; NA, not applicable. 
A

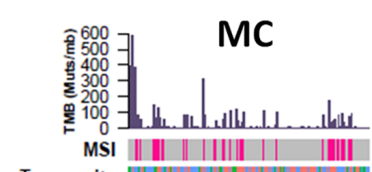

MSI $\||||||||||||||||||||| \mid$
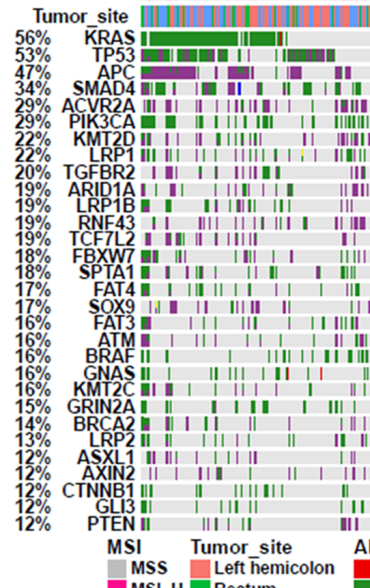

MSI Tumor_site

Rectum
Right hemi

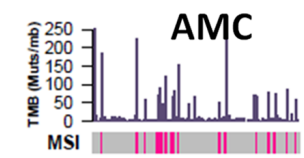

MSI || || ||||| || | || ||
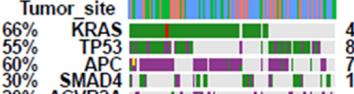

$20 \%$ ACVR2A I II III II III II $99 \%$

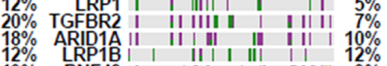

\begin{tabular}{ll|l}
$19 \%$ & RNE43 \\
$18 \%$ & TCF7L2
\end{tabular}

$\begin{array}{llll} & \\ 9 & \end{array}$

A1 || || || || ||| || || $16 \%$

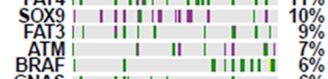

BRAF I I IIIII 6\%

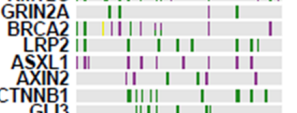

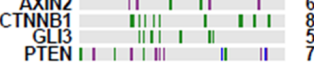

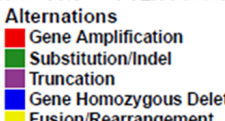

Alternations
Gene Amplification
Substitution/ndel

Substitution/In
Truncation

Gene Homozygous Delet
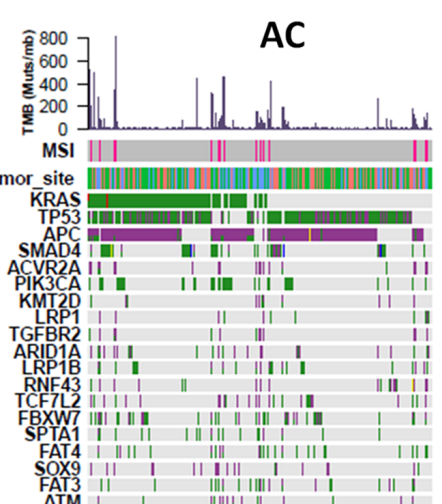

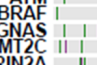

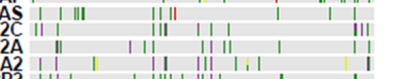

GL13

Alternations

Gene Amplification
Substitution/ndel

Truncation

Fusion/Rearrangeos Deletiont

FIGURE 1 | Comparison of common gene mutations in MC, AMC, and AC. (A) Genomic landscape showing mutated genes among MC, AMC, and AC. Each column denotes an individual tumor and each row represents the MSI status, tumor site, and individual genes. Colors indicate the type of genetic alterations as indicated in the legend. (B) Analysis of the gene alteration frequency showing a higher gene alteration

frequency in the MC group. MC, mucinous carcinoma; AMC, adenocarcinoma with mucous composition; AC, adenocarcinoma; MSI, microsatellite instability. 
(28.7 vs. $19.2 \% P=0.014)$, ACVR2A (28.7 vs. 9.1\%, $P<0.001$ ), KMT2D (22.5 vs. 6.4\%, $P<0.001)$, LRP1 (21.7 vs. $4.8 \%, P<0.001$ ), TGFBR2 (20.2 vs. 6.4\%, $P<0.001$ ), and GRIN2A (14.7 vs. $4.5 \%$, $P<0.001)$ displayed a significantly higher mutation rate in $\mathrm{MC}$ than in AC.

Furthermore, pathway analysis revealed that the mutation frequencies in MAPK, PI3K, and TGF $\beta$ pathways were higher, whereas those of cell cycle proteins and the Wnt pathway were lower, in MC and AMC than in AC (Figure 2).

\section{Comparison of Clinically Actionable Alterations Among MC, AMC, and AC}

The mutation pattern of clinically actionable alterations in $\mathrm{MC}$ was different from that in AC but similar to that in AMC. The mutation rates of BRAF V600E (10.9 vs. 3.3\%, $P<0.001)$, PIK3CA (28.7 vs. $19.2 \%, P=0.014$ ), PTEN (14.7 vs. $7.2 \%, P=0.027$ ), and BRCA2 $(17.8$ vs. $5.5 \%, P<0.001)$ were significantly higher in MC than in AC. Although HER2 mutation rates were comparable between $\mathrm{MC}$ and $\mathrm{AC}$ (3.9 vs. 6.2\%, $P=0.423)$, HER2 amplification occurred at a rate of $2.1 \%$ in AC but was not detected in MC or AMC. The mutation rate of KRAS was significantly higher in AMC than in AC (65.0 vs. $49.2 \%, P=0.001)$; however, it did not significantly differ between $\mathrm{MC}$ and $\mathrm{AC}$ or $\mathrm{MC}$ and $\mathrm{AMC}$. The mutation frequencies of clinically actionable genes in MC, AMC, and AC are summarized in Table 2.

Gene fusions in receptor tyrosine kinases have been recently identified as druggable targets in CRC (16). One patient with an MC tumor in the right colon harbored an ETV6-NTRK3 fusion and the tumor was identified as MSI-H. NCOA4-RET and FGFR2-PIBF1 fusions were observed in patients with $\mathrm{MC}$ and AMC, respectively. The frequency of druggable fusions did not significantly differ among the three CRC pathological subtypes.

\section{Comparison of Immune Biomarkers in MC, $A M C$, and $A C$}

We defined hypermutated tumors as MSI-H tumors or those harboring POLE mutations that result in a dramatic TMB elevation. In general, the proportion of hypermutated tumors was significantly higher in MC and AMC than in AC (MC 27.9\% vs. AC $8.4 \%, P<0.001$; AMC $18 \%$ vs. AC $8.4 \%, P=0.003)$.

The percentage of MSI-H tumors was significantly higher in $\mathrm{MC}$ and AMC than in AC (MC 22.5\% vs. AC 6.8\%, $P<0.001$; AMC $17 \%$ vs. AC 6.8\%, $P=0.001)$ and comparable between MC and AMC. Although the percentage of all POLE mutations among the three subtypes did not differ significantly, the proportion of POLE mutations resulting in a high TMB in MSS tumors was significantly higher in MC than in AC (5.4 vs. $1.6 \%, P=0.004)$. The median TMB and median number of somatic mutations were also significantly higher in $\mathrm{MC}$ and AMC than in AC (Figure 3 and Table 3).

\section{Hypermutated Tumors in MC}

We further evaluated the relevant immunotherapy indicators in MC, which revealed that 29 of 129 patients harbored MSI-H tumors, among which three harbored POLE mutations. Seven of 100 patients harbored MSS tumors with POLE mutations (Figure 4). Moreover, all POLE mutations detected in MSS tumors were located in the exonuclease domain, which led to extremely high levels of TMB. Only one E972G mutation in an MSS tumor was not located in the exonuclease domain and the $\mathrm{TMB}$ in this case was relatively lower $(79.5$ muts/Mb) than that in cases of POLE mutations in the exonuclease domain (TMB range, 121.1-595.5 muts/Mb). Furthermore, POLE mutations detected in three patients harboring MSI-H tumors were not present in the exonuclease domain. Details of clinical and molecular characteristics of MC with POLE mutations are summarized in Table 4.

\section{DISCUSSION}

This study identified the comprehensive genomic features of MC and AMC by targeted NGS using a large cohort of patients with CRC. We comprehensively compared genetic differences
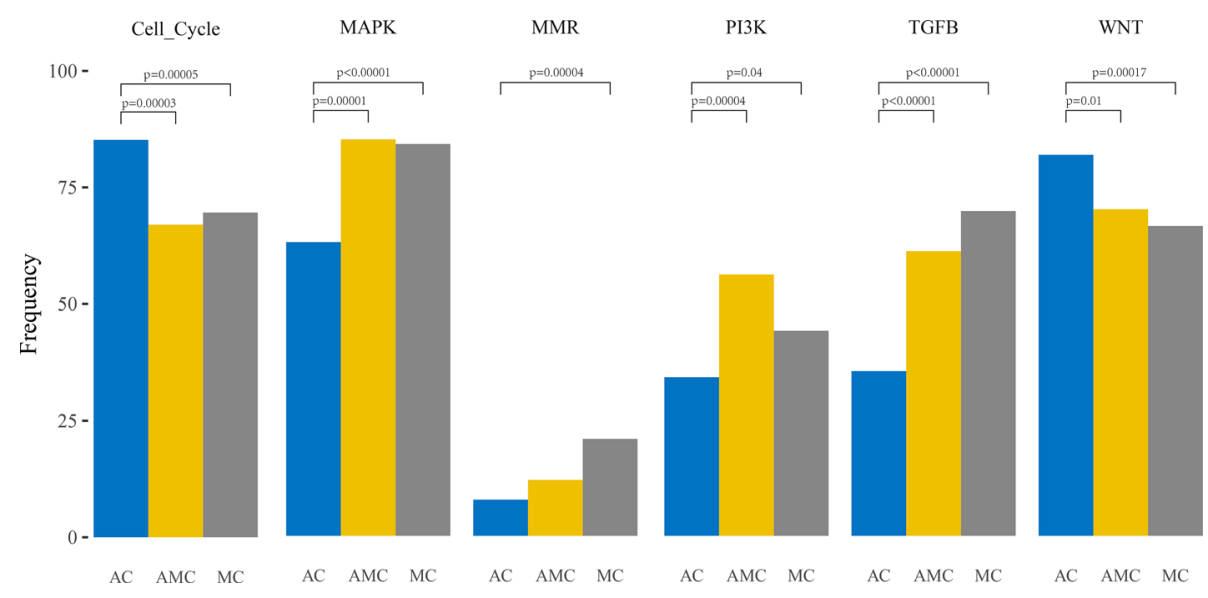

FIGURE 2 | Comparative analysis of the frequencies of genetic alterations based on signaling pathways playing an important role in oncogenesis in colorectal cancer. 
TABLE 2 | Comparison of clinically actionable gene alterations in MC, AMC, and AC.

\begin{tabular}{|c|c|c|c|c|c|c|}
\hline Genes & MCN = $129(\%)$ & AMCN = $100(\%)$ & $\mathrm{ACN}=997(\%)$ & $P$ value $M C$ vs. $A C$ & $P$ value $A M C$ vs. $A C$ & $P$ value $M C$ vs. $A M C$ \\
\hline NRAS & 4 (3.1\%) & $2(2.0 \%)$ & 36 (3.6\%) & 1.000 & 0.570 & 0.698 \\
\hline VEGFA & $2(1.6 \%)$ & $3(3.0 \%)$ & $13(1.3 \%)$ & 0.687 & 0.173 & 0.656 \\
\hline $\begin{array}{l}\text { BRAF } \\
\text { V600E }\end{array}$ & 14 (10.9\%) & 8 (8.0\%) & 33 (3.3\%) & $<0.001$ & 0.027 & 0.507 \\
\hline \multicolumn{5}{|l|}{ V600E } & & \\
\hline $\begin{array}{l}\text { BRAF } \\
\text { non-V600E }\end{array}$ & 6 (4.7\%) & 5 (5.0\%) & 22 (2.2\%) & 0.123 & 0.091 & 1.000 \\
\hline $\begin{array}{l}\text { HER2 (ERBB2) } \\
\text { mutation }\end{array}$ & 5 (3.9\%) & $4(4 \%)$ & $62(6.2 \%)$ & 0.423 & 0.508 & 1.000 \\
\hline $\begin{array}{l}\text { All druggable } \\
\text { fusion }\end{array}$ & $2(1.6 \%)$ & $1(1 \%)$ & $17(1.7 \%)$ & 1.000 & 1.000 & 1.000 \\
\hline $\begin{array}{l}\text { NTRK1 } \\
\text { fusion }\end{array}$ & $0(0 \%)$ & $0(0 \%)$ & $5(0.5 \%)$ & 0.039 & 0.689 & NA \\
\hline AKT1 & 5 (3.9\%) & 3 (3\%) & 22 (2.2\%) & 0.224 & 0.491 & 1.000 \\
\hline PTEN & 19 (14.7\%) & 14 (14\%) & 72 (7.2\%) & 0.027 & 0.013 & 0.844 \\
\hline BRCA1 & 6 (4.7\%) & 5 (5\%) & 19 (1.9\%) & 0.057 & 0.06 & 1.000 \\
\hline BRCA2 & 23 (17.8\%) & 9 (9\%) & 55 (5.5\%) & $<0.001$ & 0.043 & 0.553 \\
\hline
\end{tabular}

MC, mucinous carcinoma; AMC, adenocarcinoma with mucous composition; AC, adenocarcinoma; NA, not applicable.

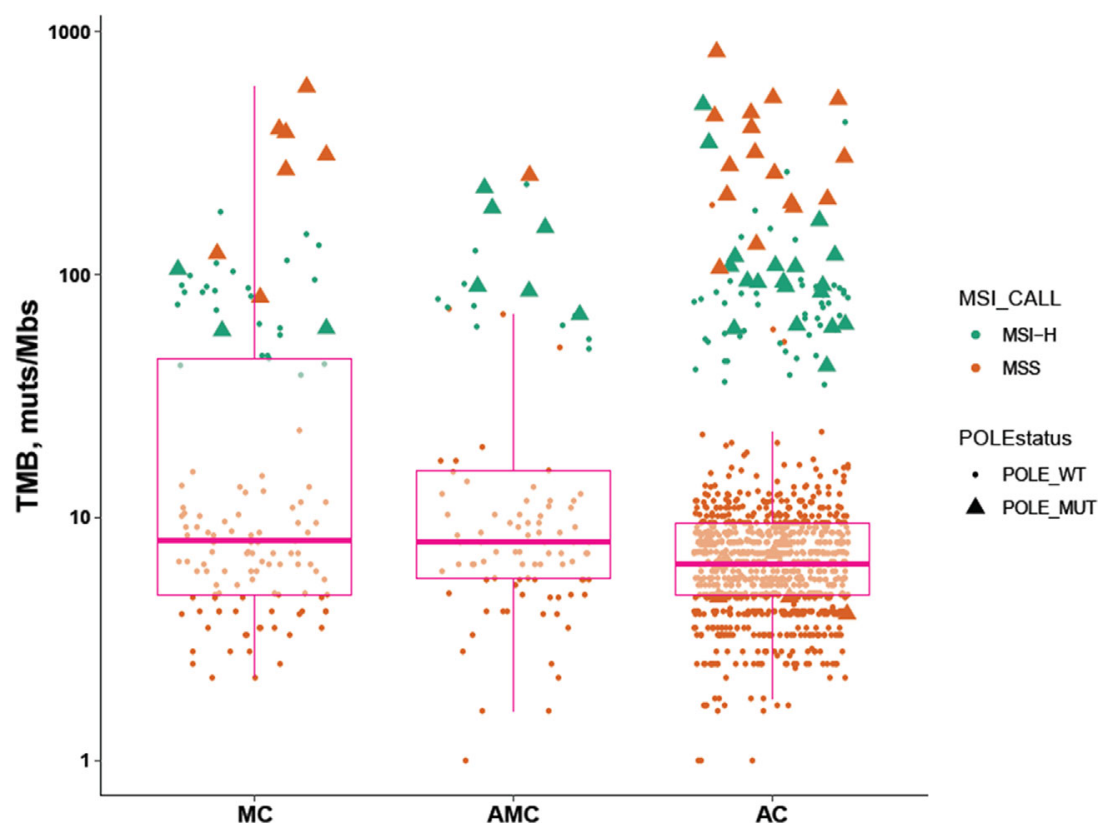

FIGURE 3 | Comparison of immunotherapy-related biomarkers in MC, AMC, and AC. MC, mucinous carcinoma; AMC, adenocarcinoma with mucous composition; $\mathrm{AC}$, adenocarcinoma; MSI, microsatellite instability; TMB, tumor mutational burden; MSS, microsatellite stability.

between $\mathrm{MC}, \mathrm{AMC}$, and $\mathrm{AC}$ and identified the following major features. In general, MC had a molecular signature that was distinct from that of AC. The genomic features were similar between AMC and MC but different between AMC and AC. MC had a distinguished mutation pattern for prevalent gene mutations and biomarkers used clinically for CRC. Most importantly, the proportion of hypermutated tumors in MC and AMC was significantly higher than that in AC, indicating the higher applicability of immunotherapy for patients with these histological subtypes. Our results support developing more 
TABLE 3 | Comparison of immunotherapy-related biomarkers in MC, AMC, and AC.

\begin{tabular}{|c|c|c|c|c|c|c|}
\hline Characteristics & $\mathrm{MCN}=129(\%)$ & $\mathrm{AMCN}=100(\%)$ & $A C N=997(\%)$ & $P$ value $M C$ vs. $A C$ & $P$ value $A M C$ vs. $A C$ & $\mathrm{P}$ value $\mathrm{MC}$ vs. AMC \\
\hline Hypermutated tumor $^{a}$ & $36(27.9)$ & $18(18.0)$ & $84(8.4)$ & $<0.001$ & 0.003 & 0.086 \\
\hline MSI-H tumor & $29(22.5)$ & $17(17.0)$ & $68(6.8)$ & $<0.001$ & 0.001 & 0.324 \\
\hline POLE & $10(7.8)$ & $7(7.0)$ & $43(4.3)$ & 0.117 & 0.209 & 1.000 \\
\hline \multicolumn{7}{|l|}{ ALL mutation } \\
\hline POLE & $7(5.4)$ & $1(1)$ & $16(1.6)$ & 0.004 & 1.000 & 0.074 \\
\hline \multicolumn{7}{|l|}{ Hypermutation in MSS tumor ${ }^{\mathrm{b}}$} \\
\hline TMB & & & & $<0.001$ & $<0.001$ & 0.967 \\
\hline Median (muts/Mb) & 7.0 & 6.9 & 5.4 & & & \\
\hline Range & $1.2-591.5$ & $0-254.7$ & 0-825.3 & & & \\
\hline Somatic mutations number & & & & 0.002 & 0.003 & 0.963 \\
\hline Median (N/tumor) & 9 & 9 & 8 & & & \\
\hline Range & $2-277$ & $1-160$ & $1-269$ & & & \\
\hline
\end{tabular}

aHypermutated tumors are defined as MSI-H tumors or tumors harboring POLE mutations, resulting in drastic TMB elevation.

${ }^{b}$ Hypermutation in MSS tumors associated with POLE-mutated cases with dramatic TMB elevation in MSS CRC, mostly caused by POLE mutations in the exonuclease domain.

MC, mucinous carcinoma; AMC, adenocarcinoma with mucous composition; AC, adenocarcinoma; CRC, colorectal cancer; MSI, microsatellite instability; TMB, tumor mutational burden; MSS, microsatellite stability.
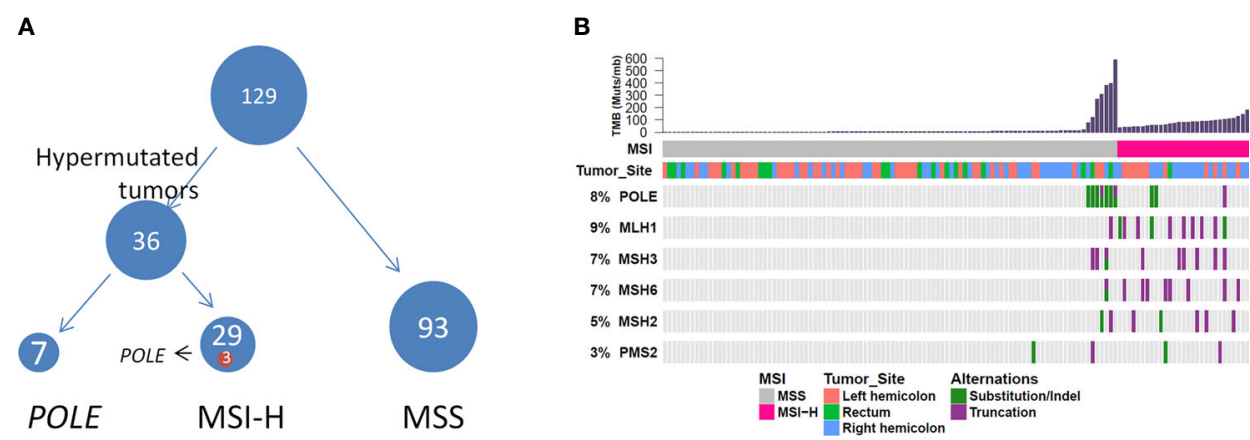

FIGURE 4 | (A) Hypermutated tumors include MSI-H tumors and tumors harboring POLE mutations. (B) Genomic landscape showing associations among MSI-H, microsatellite instability, high TMB, MSI status, and POLE mutation. TMB, tumor mutational burden.

TABLE 4 | Clinical and molecular characteristics of MC with POLE mutations.

\begin{tabular}{|c|c|c|c|c|c|c|c|c|c|c|}
\hline Number & Gene & Sex & Age(years) & Primary tumor site & Stage & TMB(muts/Mb) & MSI & Variation type & DNA change & Amino acid change \\
\hline 2 & POLE & male & 43 & Right colon & III & 58.9 & MSI-H & Substitution & $\mathrm{c} .557 \mathrm{C}>\mathrm{T}$ & p.A186V \\
\hline 4 & POLE & female & 37 & Rectum & III & 396 & MSS & Substitution & c. $1231 \mathrm{G}>\mathrm{T}$ & p.V411L \\
\hline 5 & POLE & female & 59 & Left colon & $\|$ & 268.6 & MSS & Substitution & c. $857 \mathrm{C}>\mathrm{G}$ & p.P286R \\
\hline 6 & POLE & female & 41 & Rectum & III & 121.1 & MSS & Substitution & C. $1231 G>C$ & p.V411L \\
\hline 7 & POLE & female & 56 & Right colon & $\|$ & 79.5 & MSS & Substitution & c. $2915 A>G$ & p.E972G \\
\hline 10 & POLE & male & 76 & Left colon & । & 309.5 & MSS & Substitution & c. $857 \mathrm{C}>\mathrm{G}$ & p.P286R \\
\hline
\end{tabular}

MC, mucinous carcinoma; TMB, tumor mutational burden; MSI, microsatellite instability.

tailored treatment strategies for patients with CRC according to an individual's histological subtype.

Previous studies have suggested that mutations in SMAD4, GNAS, BRAF, and KRAS occur at high frequencies in MC, whereas TP53, APC, and NRAS mutations are less common $(17,18)$. The high frequency of $B R A F$ mutations in MC is welldocumented in the literature and supported by our findings (19, 20). Patients with metastatic CRC harboring a BRAF V600E mutation have a significantly worse prognosis. This study found the BRAF V600E mutation rate was significantly higher in MC and AMC than in AC, whereas the mutation rate of BRAF (nonV600E) did not significantly differ among the three groups. The SMAD4 mutation frequency was significantly higher in MC and AMC than in AC. Patients with a SMAD4 deletion have worse relapse-free survival and are resistant to chemotherapy with 5fluorouracil (21). MCs were associated with an unsatisfactory 
response to neoadjuvant chemotherapy. However, whether SMAD4 plays a role in chemotherapy resistance mechanisms needs further research. On the other hand, the stage of the cancer is significantly associated with the frequency of specific mutation. For example, BRAF (V600E) is more frequent in high-stage MC. The observation suggests that the variation in the mutation rates among the three cancer types is attributed to the different clonal evolution processes, from which MC arises as a unique subtype.

A recent study reported that approximately $5 \%$ of patients with CRC harbor a HER2 mutation. In patients with CRC, nearly half of HER2 alterations are mutation rather than amplification or protein overexpression. Herein, HER2 amplification was not observed in MC. However, a proportion of patients with MC harbored HER2 mutations. Previous animal experiments have reported that the growth of implanted tumors harboring mutant HER 2 can be inhibited by HER2 inhibitors, including trastuzumab, lapatinib, and afatinib, alone and in combination with trastuzumab and tyrosine kinase inhibitors (22-24).

Immune checkpoint inhibitors (ICIs) have recently been widely used in solid and hematological malignancies (25). We defined hypermutated tumors as MSI-H tumors or those harboring POLE mutations that result in a dramatic TMB elevation, as there is robust evidence for MSI-H and POLE mutations as predictive biomarkers for a good response to immunotherapy in CRC $(26,27)$. Pembrolizumab has been approved for treating solid tumors with MSI-H/deficient mismatch repair $(\mathrm{dMMR})$ and nivolumab \pm ipilimumab has been approved for treating advanced CRC with MSI-H/dMMR $(28,29)$. Recently, a study on neoadjuvant treatment of CRC was conducted using a combinatorial treatment with an anti-PD-1 antibody and anti-CTLA-4 antibody. The treatment resulted in a pathological response in $20 / 20$ patients and primary pathological remission in $19 / 20$ patients with dMMR tumors (30). MC is significantly more likely to be associated with MSI-H in the colon and rectum (20). The proportion of MSI-H tumors in this study was significantly higher in $\mathrm{MC}$ and $\mathrm{AMC}$ than in $\mathrm{AC}$, suggesting that immunotherapy is suitable for a larger proportion of patients with $\mathrm{MC}$ and $\mathrm{AMC}$. MC was more prevalent in stage III CRC in this study, indicating that patients are more likely to develop local lymph node metastasis and present locally advanced CRC. In some cases of locally advanced CRC, it is challenging for surgeons to perform R0 (margin-negative) resection, which results in a worse prognosis for patients. ICIs in a neoadjuvant setting would be an effective treatment alternative for patients with MC with MSI-H/dMMR; thus, it is necessary to clarify the MSI/MMR status before any treatment.

Immunotherapy in MSS CRC tumors still lacks efficacy; therefore, there is an urgent need to identify biomarkers for immunotherapy in MSS tumors. Hypermutation in MSS CRCs is often associated with POLE mutations accompanied by dramatic TMB elevation, owing to the loss of DNA replication fidelity caused by POLE mutations in the exonuclease domain (27). Wang et al. summarized the POLE/POLD1 mutation rate in 47,721 patients with different cancer types and identified that patients harboring POLE/POLD1 mutations have a significantly higher TMB. When adjusting for cancer types and MSI status for multivariate Cox regression analysis, POLE/POLD1 mutations were found to be independent risk factors for identifying patients that could benefit from ICI treatment (27). In this study, the frequency of POLE mutations resulting in high TMB in MSS tumors was significantly higher in MC than in AC. In addition, the proportion of hypermutated tumors (MSI-H or POLE mutations) was $27.9 \%$ in MC, suggesting that up to $30 \%$ of patients with CRC MC may benefit from immunotherapy. Furthermore, the mutation pattern of POLE differed between MSS and MSI-H tumors and POLE mutations occurring in the exonuclease domain markedly increased the TMB in MSS tumors.

We acknowledge that the current study had several limitations. First, the retrospective study design could not exclude selection bias. Second, the clinical data of patients' treatments and outcomes were not controlled and collected in the current study; therefore, the clinical impacts of our findings need further confirmation. Finally, on the potential benefit of ICI, the effects of tumor infiltrating lymphocytes need to be evaluated along with the mutational profile as well as MSI and POLE statuses; whereas in the current study still lacks the pathological data for tumor immune microenvironment. On the other hand, the somatic mutational landscape is also affected by the host immune environment, hence serve as a proxy to the activities of the immune cells.

In spite of the limitations, using the large cohorts of MC ( $n=$ $129)$ and AMC $(n=100)$ via a comprehensive targeted NGS panel, our results reveal the molecular landscapes of MC, AMC, and AC, which could lead to tailored treatment for different histological subtypes of CRC. The selection of baseline clinical and pathological characteristics was relatively intact in this study, allowing the analysis of clinical and genomic features. Our findings shed new light on the treatment and management of patients with MC and AMC. Further prospective studies in patients with $\mathrm{MC}$ and $\mathrm{AMC}$ are warranted to validate our findings, especially regarding the potential use of immunotherapy.

\section{CONCLUSIONS}

We identified a distinct genomic landscape in colorectal MC via comprehensive genomic profiling for commonly mutated and clinically actionable genes. Hypermutated tumors account for nearly $30 \%$ of $\mathrm{MC}$, suggesting that a large proportion of patients with MC may benefit from immunotherapy; therefore, there is a need for comprehensive molecular testing in these patients. AMC has similar genomic features to MC but different from $\mathrm{AC}$, suggesting the potential for the use of $\mathrm{MC}$ treatment strategies for treating AMC.

\section{DATA AVAILABILITY STATEMENT}

The data presented in the study are deposited in the CNGB Sequence Archive, repository, accession number is CNP0001753. 
The reviewer link is http://db.cngb.org/cnsa/review/show/ CNP0001753_20210422_a35ddad7.

\section{ETHICS STATEMENT}

The studies involving human participants were reviewed and approved by Institution Review Board of the Sixth Affiliated Hospital of Sun Yat-Sen University. The patients/participants provided their written informed consent to participate in this study.

\section{AUTHOR CONTRIBUTIONS}

Study design: LH, LK, SZ, and TL. Study recruitment, clinical sample, and data acquisition: XZ, YC, FX, HH, ZZ, SZ, and TL. Bioinformatic analysis: WW and FW. Primary results interpretation: LH and SL. Manuscript drafting: SL, LH, LK, and SZ. All authors contributed to the article and approved the submitted version.

\section{REFERENCES}

1. Bray F, Ferlay J, Soerjomataram I, Siegel RL, Torre LA, Jemal A. Global Cancer Statistics 2018: GLOBOCAN Estimates of Incidence and Mortality Worldwide for 36 Cancers in 185 Countries. CA Cancer J Clin (2018) 68:394424. doi: 10.3322/caac. 21492

2. Dekker E, Tanis PJ, Vleugels JLA, Kasi PM, Wallace MB. Colorectal Cancer. Lancet (2019) 394:1467-80. doi: 10.1016/S0140-6736(19)32319-0

3. Hugen N, Brown G, Glynne-Jones R, de Wilt JH, Nagtegaal ID. Advances in the Care of Patients With Mucinous Colorectal Cancer. Nat Rev Clin Oncol (2016) 13:361-9. doi: 10.1038/nrclinonc.2015.140

4. Luo C, Cen S, Ding G, Wu W. Mucinous Colorectal Adenocarcinoma: Clinical Pathology and Treatment Options. Cancer Commun (Lond) (2019) 39:13. doi: 10.1186/s40880-019-0361-0

5. Hugen N, van Beek JJ, de Wilt JH, Nagtegaal ID. Insight Into Mucinous Colorectal Carcinoma: Clues From Etiology. Ann Surg Oncol (2014) 21:296370. doi: 10.1245/s10434-014-3706-6

6. Catalano V, Loupakis F, Graziano F, Torresi U, Bisonni R, Mari D, et al. Mucinous Histology Predicts for Poor Response Rate and Overall Survival of Patients With Colorectal Cancer and Treated With First-Line Oxaliplatinand/or Irinotecan-Based Chemotherapy. Br J Cancer (2009) 100:881-7. doi: 10.1038/sj.bjc.6604955

7. Li C, Zheng H, Jia H, Huang D, Gu W, Cai S, et al. Prognosis of Three Histological Subtypes of Colorectal Adenocarcinoma: A Retrospective Analysis of 8005 Chinese Patients. Cancer Med (2019) 8:3411-9. doi: $10.1002 / \mathrm{cam} 4.2234$

8. Simha V, Kapoor R, Gupta R, Bahl A, Nada R. Mucinous Adenocarcinoma of the Rectum: A Poor Candidate for Neo-Adjuvant Chemoradiation? J Gastrointest Oncol (2014) 5:276-9. doi: 10.3978/j.issn.2078-6891.2014.020

9. Moretto R, Morano F, Ongaro E, Rossini D, Pietrantonio F, Casagrande M, et al. Lack of Benefit From Anti-EGFR Treatment in RAS and BRAF WildType Metastatic Colorectal Cancer With Mucinous Histology or Mucinous Component. Clin Colorectal Cancer (2019) 18:116-24. doi: 10.1016/ j.clcc.2019.02.007

10. Hugen N, Simons M, Halilovic A, van der Post RS, Bogers AJ, Marijnissen-van Zanten MA, et al. The Molecular Background of Mucinous Carcinoma Beyond MUC2. J Pathol Clin Res (2015) 1:3-17. doi: 10.1002/cjp2.1

11. Verhulst J, Ferdinande L, Demetter P, Ceelen W. Mucinous Subtype as Prognostic Factor in Colorectal Cancer: A Systematic Review and MetaAnalysis. J Clin Pathol (2012) 65:381-8. doi: 10.1136/jclinpath-2011-200340

12. Ogino S, Brahmandam M, Cantor M, Namgyal C, Kawasaki T, Kirkner G, et al. Distinct Molecular Features of Colorectal Carcinoma With Signet Ring

\section{FUNDING}

This study was supported by the Fundamental Research Funds for the Central Universities, grant number 16ykjc25, Sun Yat-Sen University Clinical Research 5010 Program, grant number 2016005 and Province Natural Science Fund of Guangdong (2018A030313621).

\section{ACKNOWLEDGMENTS}

We thank Jian Wang and Jicheng Yao from OrigiMed for their contributions to the bioinformatic analysis.

\section{SUPPLEMENTARY MATERIAL}

The Supplementary Material for this article can be found online at: https://www.frontiersin.org/articles/10.3389/fonc.2021.603564/ full\#supplementary-material

Cell Component and Colorectal Carcinoma With Mucinous Component. Mod Pathol (2006) 19:59-68. doi: 10.1038/modpathol.3800482

13. Tozawa E, Ajioka Y, Watanabe H, Nishikura K, Mukai G, Suda T, et al. Practice, Mucin Expression, p53 Overexpression, and Peritumoral Lymphocytic Infiltration of Advanced Colorectal Carcinoma With Mucus Component: Is Mucinous Carcinoma a Distinct Histological Entity? Pathol Res Pract (2007) 203:567-74. doi: 10.1016/j.prp.2007.04.013

14. Cao J, Chen L, Li H, Chen H, Yao J, Mu S, et al. An Accurate and Comprehensive Clinical Sequencing Assay for Cancer Targeted and Immunotherapies. Oncologist (2019) 24:e1294-302. doi: 10.1634/theoncologist.2019-0236

15. Chalmers ZR, Connelly CF, Fabrizio D, Gay L, Ali SM, Ennis R, et al. Analysis of 100,000 Human Cancer Genomes Reveals the Landscape of Tumor Mutational Burden. Genome Med (2017) 9:34. doi: 10.1186/s13073-017-0424-2

16. Garcia-Aranda M, Redondo M. Targeting Receptor Kinases in Colorectal Cancer. Cancers (Basel) (2019) 11:433-57. doi: 10.3390/cancers11040433

17. Khan M, Loree JM, Advani SM, Ning J, Li W, Pereira AAL, et al. Prognostic Implications of Mucinous Differentiation in Metastatic Colorectal Carcinoma can Be Explained by Distinct Molecular and Clinicopathologic Characteristics. Clin Colorectal Cancer (2018) 17:e699-709. doi: 10.1016/j.clcc.2018.07.005

18. Kim H, Kim BH, Lee D, Shin E. Genomic Alterations in Signet Ring and Mucinous Patterned Colorectal Carcinoma. Pathol Res Pract (2019) 215:152566. doi: 10.1016/j.prp.2019.152566

19. Tanaka H, Deng G, Matsuzaki K, Kakar S, Kim GE, Miura S, et al. BRAF Mutation, CpG Island Methylator Phenotype and Microsatellite Instability Occur More Frequently and Concordantly in Mucinous Than non-Mucinous Colorectal Cancer. Int J Cancer (2006) 118:2765-71. doi: 10.1002/ijc.21701

20. Reynolds IS, Furney SJ, Kay EW, McNamara DA, Prehn JHM, Burke JP. Meta-Analysis of the Molecular Associations of Mucinous Colorectal Cancer. Br J Surg (2019) 106:682-91. doi: 10.1002/bjs.11142

21. Wasserman I, Lee LH, Ogino S, Marco MR, Wu C, Chen X, et al. Smad4 Loss in Colorectal Cancer Patients Correlates With Recurrence, Loss of Immune Infiltrate, and Chemoresistance. Clin Cancer Res (2019) 25:1948-56. doi: 10.1158/1078-0432.CCR-18-1726

22. Greally M, Kelly CM, Cercek A. Her2: An Emerging Target in Colorectal Cancer. Curr Probl Cancer (2018) 42:560-71. doi: 10.1016/j.currproblcancer.2018.07.001

23. Siena S, Sartore-Bianchi A, Marsoni S, Hurwitz HI, McCall SJ, Penault-Llorca F, et al. Targeting the Human Epidermal Growth Factor Receptor 2 (HER2) Oncogene in Colorectal Cancer. Ann Oncol (2018) 29:1108-19. doi: 10.1093/annonc/mdy100

24. Cheng Z, Shao X, Xu M, Wang J, Kuai X, Zhang L, et al. Rab1A Promotes Proliferation and Migration Abilities Via Regulation of the HER2/AKTindependent mTOR/S6K1 Pathway in Colorectal Cancer. Oncol Rep (2019) 41:2717-28. doi: 10.3892/or.2019.7071 
25. Gibney GT, Weiner LM, Atkins MB. Predictive Biomarkers for Checkpoint Inhibitor-Based Immunotherapy. Lancet Oncol (2016) 17:e542-51. doi: 10.1016/S1470-2045(16)30406-5

26. Schrock AB, Ouyang C, Sandhu J, Sokol E, Jin D, Ross JS, et al. Tumor Mutational Burden is Predictive of Response to Immune Checkpoint Inhibitors in MSI-high Metastatic Colorectal Cancer. Ann Oncol (2019) 30:1096-103. doi: 10.1093/annonc/mdz134

27. Wang F, Zhao Q, Wang YN, Jin Y, He MM, Liu ZX, et al. Evaluation of POLE and POLD1 Mutations as Biomarkers for Immunotherapy Outcomes Across Multiple Cancer Types. JAMA Oncol (2019) 5(10):1504-6. doi: 10.1001/ jamaoncol.2019.2963

28. Le DT, Durham JN, Smith KN, Wang H, Bartlett BR, Aulakh LK, et al. Mismatch Repair Deficiency Predicts Response of Solid Tumors to PD-1 Blockade. Science (2017) 357:409-13. doi: 10.1126/science.aan6733

29. Overman MJ, Lonardi S, Wong KYM, Lenz HJ, Gelsomino F, Aglietta M, et al. Durable Clinical Benefit With Nivolumab Plus Ipilimumab in DNA Mismatch Repair-Deficient/Microsatellite Instability-High Metastatic Colorectal Cancer. J Clin Oncol (2018) 36:773-9. doi: 10.1200/JCO.2017.76.9901
30. Chalabi M, Fanchi LF, Dijkstra KK, Van den Berg JG, Aalbers AG, Sikorska K, et al. Neoadjuvant Immunotherapy Leads to Pathological Responses in MMRproficient and MMR-deficient Early-Stage Colon Cancers. Nat Med (2020) 26:566-76. doi: 10.1038/s41591-020-0805-8

Conflict of Interest: TL, FW and WW were employed by OrigiMed.

The remaining authors declare that the research was conducted in the absence of any commercial or financial relationships that could be construed as a potential conflict of interest.

Copyright (c) 2021 Huang, Luo, Zhang, Cai, Xue, Hu, Zeng, Lin, Wang, Wang, Zhang and Kang. This is an open-access article distributed under the terms of the Creative Commons Attribution License (CC BY). The use, distribution or reproduction in other forums is permitted, provided the original author(s) and the copyright owner(s) are credited and that the original publication in this journal is cited, in accordance with accepted academic practice. No use, distribution or reproduction is permitted which does not comply with these terms. 